\title{
Aberrant KLK4 gene promoter hypomethylation in pediatric hepatoblastomas
}

\author{
BAIHUI LIU* ${ }^{*}$ XIMAO CUI ${ }^{*}$, SHAN ZHENG, KUIRAN DONG and RUI DONG \\ Department of Pediatric Surgery, Children's Hospital of Fudan University, \\ Shanghai Key Laboratory of Birth Defects and Key Laboratory of Neonatal Disease, \\ Ministry of Health, Shanghai 201102, P.R. China
}

Received June 16, 2015; Accepted October 24, 2016

DOI: $10.3892 / \mathrm{ol} .2017 .5558$

\begin{abstract}
DNA methylation has a crucial role in cancer biology and has been recognized as an activator of oncogenes and inactivator of tumor suppressor genes, both of which are mechanisms for tumorigenesis. Kallikrein-related peptidase 4 (KLK4), has been suggested to be an oncogene in various types of cancer. The aim of the present study was to assess the DNA methylation patterns of the KLK4 gene in cancerous samples harvested from patients with hepatoblastoma (HB). KLK4 mRNA expression levels were detected using reverse transcription-quantitative polymerase chain reaction and assessed its DNA methylation patterns using high-throughput mass spectrometry on a matrix-assisted laser desorption/ionization time-of-flight mass array. A total of $10 \mathrm{HB}$ and 10 normal liver tissue samples were obtained from patients with HB. The results of the present study showed that a significantly higher level of KLK4 mRNA expression levels were detected in HB tissues, as compared with the matched controls. Furthermore, the KLK4 gene promoter region was distinctively less methylated in the HB samples compared with the controls and negatively correlated with KLK4 mRNA expression levels. These findings indicate that aberrant methylation of KLK4 may contribute to its upregulated mRNA expression in HB.
\end{abstract}

Correspondence to: Mr. Kuiran Dong or Mr. Rui Dong, Department of Pediatric Surgery, Children's Hospital of Fudan University, Shanghai Key Laboratory of Birth Defects and Key Laboratory of Neonatal Disease, Ministry of Health, 399 Wan Yuan Road, Shanghai 201102, P.R. China

E-mail: kuirand@hotmail.com

E-mail: dongrui_1982@126.com

*Contributed equally

Key words: DNA methylation, kallikrein-related peptidase 4, matrix-assisted laser desorption/ionization time-of-flight, hepatoblastoma

\section{Introduction}

Hepatoblastoma (HB) is the most common type of malignant liver tumor in infants and children. Although it accounts for just $0.5-1.5$ cases per million children per year, the mortality rate is $35-50 \%$ in high-risk patients (1). Previous studies have suggested an association with familial adenomatous polyposis (FAP) (2), both low and high birth weights (3), and constitutional trisomy 18 (4); however its etiology remains unknown.

Currently, alphafetoprotein levels, histological analysis, tumor resectability and tumor metastasis are the only prognosis factors for HB. Therefore, novel targets for early detection and improved therapies and prognosis are required.

Epigenetic reprogramming has a crucial role in tumorigenesis; one of the key mechanisms is DNA methylation. DNA methylation, maintained by DNA methyltransferases, refers to the addition of a methyl group to the carbon 5 of the cytosine ring in $\mathrm{CpG}$ dinucleotides of gene promoter regions (5). DNA methylation is the only genetically programmed DNA modification process in mammals that is involved in the regulation of various biological processes, including gene transcription, X-chromosome inactivation, genomic imprinting and chromatin modification (6-8). It is generally recognized that DNA methylation is a form of gene closure; the higher the level of methylation, the more silent a gene is. Therefore, the hypermethylation of suppressor genes or hypomethylation of oncogenes may contribute to tumorigenesis. This hypothesis has been investigated and supported in various studies on tumors (9), including HB (10).

Kallikrein-related peptidase 4 (KLK4), which is a member of the KLK family, is considered to be an oncogene associated with various types of cancer, including colorectal adenocarcinoma and prostate cancer $(11,12)$. In a pilot study, we detected aberrant expression of KLK4 in HB and normal tissues, and theorized if alterations in methylation status affect its expression. Therefore, the present study aimed to investigate KLK4 methylation in $10 \mathrm{HB}$ samples in order to profile genes that are differentially methylated in this disease.

\section{Materials and methods}

Patients and sample collection. The present study utilized $10 \mathrm{HB}$ and adjacent non-tumor tissues from patients who 
Table I. Clinical and pathological characteristics of the study subjects.

\begin{tabular}{ccccc}
\hline Subject & Age $($ months) & Diagnosis type & Alpha-fetoprotein (ng/ml) \\
\hline 1 & 7 & Gender & Mixed embryonal/fetal subtype & 68490 \\
2 & 23 & Male & Mixed embryonal/fetal subtype & $>121,000$ \\
3 & 11 & Female & Mixed embryonal/fetal subtype & $>121,000$ \\
4 & Female & Mixed embryonal/fetal subtype & $>121,000$ \\
5 & 10 & Female & Mixed embryonal/fetal subtype & $>121,000$ \\
6 & 20 & Male & Mixed embryonal/fetal subtype & $>121,000$ \\
7 & 7 & Male & Epithelial type & $>121,000$ \\
8 & 30 & Male & Epithelial type & $>121,000$ \\
9 & 14 & Male & Epithelial type & $>121,000$ \\
10 & 19 & Female & Epithelial type & $>121,000$ \\
\hline
\end{tabular}

${ }^{\mathrm{a}} \mathrm{On}$ the date of serum sample collection.

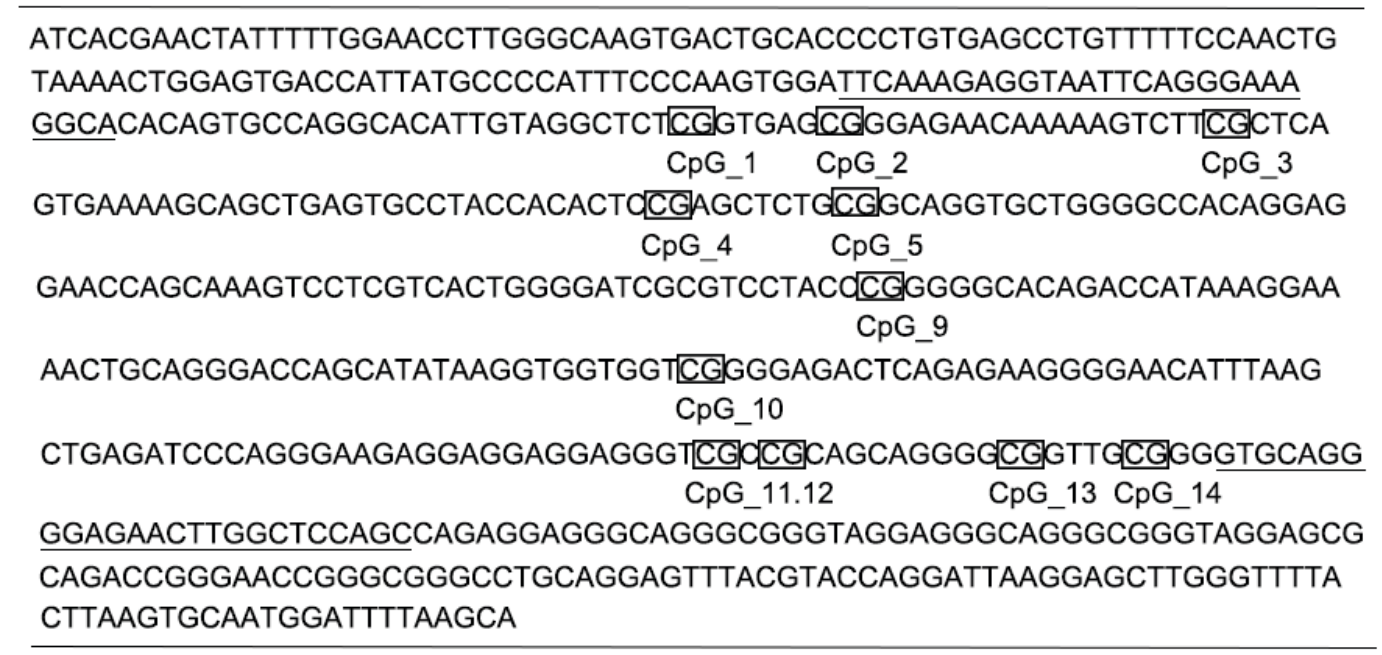

Figure 1. DNA sequence of the kallikrein-related peptidase 4 promoter. Primers are underlined.

underwent partial hepatectomy at the Children's Hospital of Fudan University (Shanghai, China). The patients ranged in age from 7 to 30 months. Informed consent was obtained retrospectively from clinical files and the diagnoses were confirmed by the Department of Pathology following the presence of $>80 \%$ viable tumor cells (13). Clinical and pathological data are listed in Table I. The Ethics Committee of the Children's Hospital of Fudan University approved the use of these human samples. Genomic DNA was extracted according to standard procedures from 10 matched $\mathrm{HB}$ tumors and non-tumor tissue sections that were stored at $-80^{\circ} \mathrm{C}$ immediately after surgery.

DNA/RNA extraction and reverse transcription quantitative-polymerase chain reaction ( $R T-q P C R)$. DNA was extracted from $10 \mathrm{HB}$ primary tumors and adjacent non-tumor tissues. DNA samples were stored at $-80^{\circ} \mathrm{C}$ until subsequent use for mass spectrometry analysis. Total RNA was extracted from $10 \mathrm{HB}$ tumor and non-tumor pairs using TRIzol reagent (Invitrogen; Thermo Fisher Scientific,
Inc., Waltham, MA, USA), and, reverse-transcribed into cDNA using a PrimeScript RT reagent kit (Perfect Real Time) with gDNA Eraser (Takara Biotechnology Co., Ltd., Dalian, China) following the manufacturer's protocol. This was then subjected to qPCR analysis to assess the levels of KLK4 mRNA expression using a SYBRGreen PCR kit (Takara Biotechnology Co., Ltd.). The KLK4 primers used for qPCR were: Forward, 5'-GGACTCCTGCAACGGTGA CTCT-3' and reverse, 5'-TAGACACCTGGCACGCCAACT T-3'. The PCR cycling conditions were as follows: $95^{\circ} \mathrm{C}$ for $30 \mathrm{sec}$, followed by $95^{\circ} \mathrm{C}$ for $5 \mathrm{sec}$ and $60^{\circ} \mathrm{C}$ for $34 \mathrm{sec}$, for 45 cycles. For quantitative results, KLK4 expression was represented as fold-change by the $2^{-\Delta \Delta \mathrm{Cq}}$ method and statistically analyzed (14).

Primer design and PCR tagging for EpiTYPER assay. Primers for the KLK4 gene were designed to cover the regions with the most $\mathrm{CpG}$ sites (Fig. 1). Selected amplicons were $500 \mathrm{bp}$ upstream of the promoter of the KLK4 gene. Genomic DNA was bisulfite-treated, and its reverse primer 


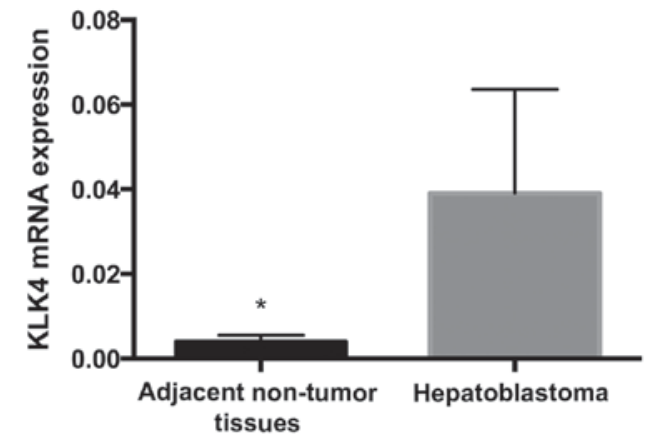

Figure 2. KLK4 mRNA expression levels were significantly greater in the HB samples, as compared to adjacent non-tumor tissues $\left({ }^{*} \mathrm{P}<0.01\right)$. KLK4, kallikrein-related peptidase 4.

was supplemented with a T7-promoter tag for subsequent PCR amplification.

Mass spectrometry. Following treatment with shrimp alkaline phosphatase (SAP) in vitro transcription and uracil-specific cleavage, the DNA samples were robotically dispensed onto silicon matrix preloaded chips (SpectroCHIP; Sequenom, San Diego, CA, USA). Mass spectra were collected using a MassARRAY Compact matrix-assisted laser desorption/ionization time-of-flight system (Sequenom), and the methylation ratios of the spectra were generated using EpiTYPER software v1.0 (Sequenom).

Statistical methods. Statistical analyses and graphical depiction of data were generated using GraphPad Prism 5.0 (GraphPad Software, Inc., La Jolla, CA, USA). Results are presented as the mean \pm standard error of the mean and were evaluated using a two-tailed Student's t-test unless otherwise specified, in which instance unpaired t-test or Pearson's correlation analysis were used. Statistical analyses were also performed using SPSS 19.0 software (IBM SPSS, Armonk, NY, USA) for Windows. $\mathrm{P}<0.05$ was considered statistically significant to indicate a statistically significant difference.

\section{Results}

KLK4 mRNA expression levels are elevated in HB. Expression levels of KLK4 mRNA were analyzed in the 10 pairs of tumor $(\mathrm{n}=10)$ and adjacent normal liver $(\mathrm{n}=10)$ tissues using RT-qPCR. Expression levels of KLK4 in HB tissues were significantly increased, as compared with matched non-tumor liver tissues $(0.039 \pm 0.0077$ vs. $0.004 \pm 0.0005$, respectively; $\mathrm{P}<0.01$; Fig. 2).

DNA methylation of KLK4 is reduced in $H B$. Methylation patterns of KLK4 were verified in the $10 \mathrm{HB}$ samples and match non-tumor controls. Enriched methylation positions located at $500 \mathrm{bp}$ upstream of the transcription initiation site were analyzed; 11/22 sites in the amplicons were amenable to analysis. Using two-way hierarchical cluster analysis, two sites were found to be at a significantly lower degree of methylation in the HB tissues, as compared with their non-tumor counterparts (Fig. 3). Furthermore, these sites exhibited a significant difference in the mean methylation levels of the
KLK4 gene between HB and adjacent non-tumor tissues $(0.3755 \pm 0.03218$ vs. $0.4885 \pm 0.01176$, respectively; $\mathrm{P}<0.01$; Fig. 4).

Correlation between hypomethylation with KLK4 and expression of $m R N A$. Using linear Pearson's R correlation, the correlations between KLK4 mRNA expression and DNA methylation status of $\mathrm{CpG} 4$ and $\mathrm{CpG} 9$ sites harvested from the 10 samples were analyzed. This analysis demonstrated that the expression of KLK4 is negatively correlated with its methylation status ( $r=-0.47$; $\mathrm{P}=0.037$; Fig. 5).

\section{Discussion}

Pediatric solid tumors develop after relatively short latency periods, and thus are becoming one of the most common reasons for child mortality $(15,16)$. HB is the most common liver tumor found in children, and its incidence is increasing in North America and Europe (17). Although its pathogenesis and progression have been extensively studied for the last two decades, its etiology remains to be fully elucidated. Through a previous study that involved sequencing of the HB exome, we recently detected a novel oncogene (caprin family member 2) and three tumor suppressors (speckle-type POZ protein, olfactory receptor family 5 subfamily I member 1 and cell division cycle 20B) that influence HB cell growth (18). The present group has also detected an association between long non-coding RNA (lncRNA) and HB in a previous study (19).

Hypermethylation or hypomethylation of gene promoter regions is recognized as one of the mechanisms that can silence or activate the oncogenes (20). It has recently been indicated that aberrant promoter methylation of the RAS association domain family protein 1 may contribute to the pathogenesis of $\mathrm{HB}$ and is considered to be a significant prognostic indicator in $\operatorname{HB}(1,21)$.

KLK4 is a member of the KLK family. It has previously been reported that KLK4 is overexpressed in endometrial carcinoma (22), colorectal adenocarcinoma, and prostate cancer; however, the mechanism remains unclear. Detection of KLK4 mRNA expression levels in HB and normal liver tissues by qPCR in the present study demonstrated a significant difference between tissue types. KLK4 mRNA expression levels in HB tissues were significantly higher than in normal liver tissues. In an independent cohort of 10 adjacent HB-non-tumor tissues pairs, we theorized whether there was a correlation between KLK4 mRNA expression and DNA methylation. To further understand the association between HB and KLK4 methylation, the methylation status of KLK4 genes were analyzed in the 10 paired samples. Following methylation quantification of the KLK4 gene using MALDI-TOF MS, three CpG sites were detected per sample. Hypermethylation degrees of two CpG sites in the promoter regions of the KLK4 gene for normal liver tissues were significantly higher than those of HB samples. Furthermore, correlation analysis indicated that expression of KLK4 mRNA is significantly negatively correlated with its methylation status. This finding supports previously published data, which suggested that KLK4 may be an oncogene (23-26). 

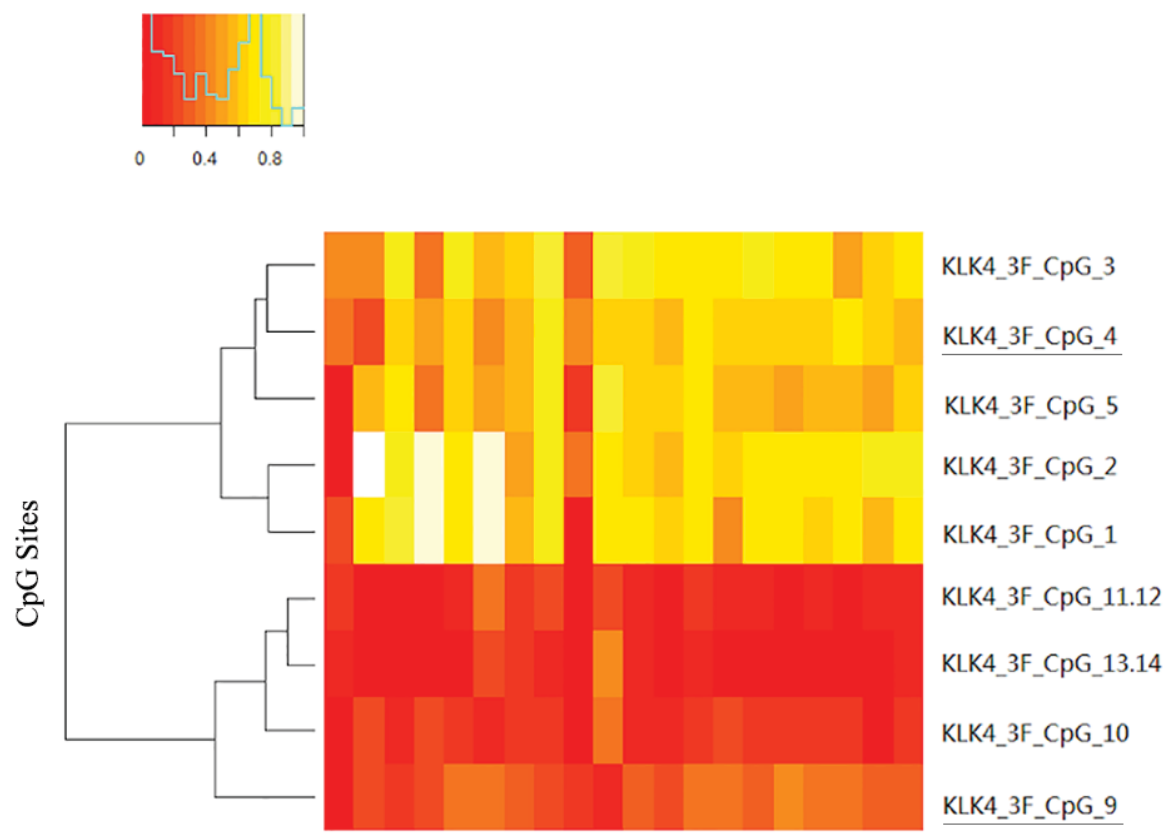

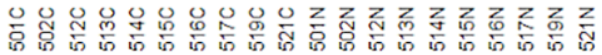

Samples

Figure 3. Two-way hierarchical cluster analysis of methylation patterns between 10 hepatoblastoma and adjacent non-tumor tissues. The two aberrant methylation sites are underlined. Red clusters indicate $0 \%$ methylated, whereas yellow clusters indicate $100 \%$ methylated. The color gradient between red and yellow indicates methylation ranging from 0 to 100, and white clusters indicate CpG sites that were not analyzed. KLK4, kallikrein-related peptidase 4.

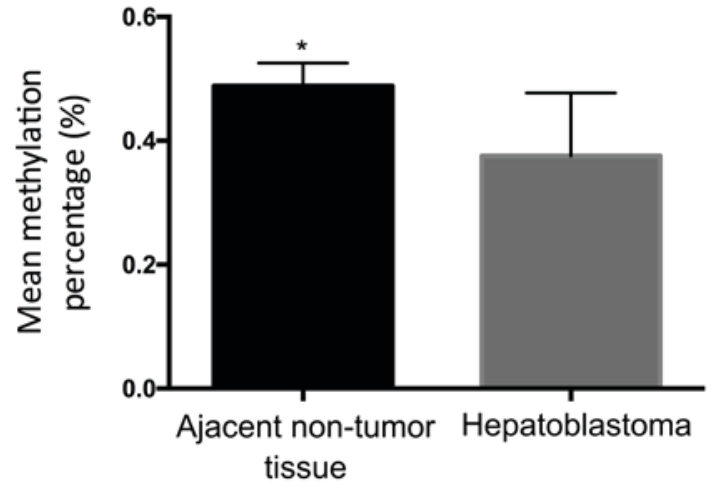

Figure 4. Methylation levels of KLK4 were significantly reduced in the hepatoblastoma samples $(\mathrm{n}=10)$, as compared with adjacent non-tumor tissues $($ P $<0.01)$. KLK4, kallikrein-related peptidase 4.

To the best of our knowledge, the present study is the first to examine the methylation of KLK4 genes in HB, and an association between the level of KLK4 mRNA and methylation was successfully detected. This finding is consistent with the results of previous studies, demonstrating that hypomethylation of promoter regions of crucial genes is able to activate relevant gene expression and may contribute to tumorigenesis (27-29). A limitation of the present study is that only 10 pairs of patient samples were used. Additional samples are required for investigation in order to more accurately represent the population.

In conclusion, the present findings indicate that aberrant promoter methylation of KLK4 may contribute to the tumorigenesis of $\mathrm{HB}$, and that KLK4 may be a potential biomarker for HB. However, since HB is an uncommon disease, its

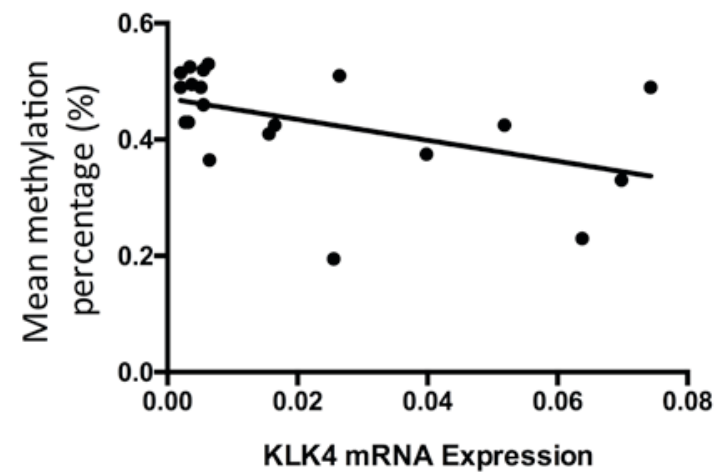

Figure 5. Correlation analysis of KLK4 mRNA expression and the percentage of DNA methylation in 10 hepatoblastoma primary tissues (two-tailed Pearson Correlation: $\mathrm{r}=-0.47 ; \mathrm{P}=0.037)$. KLK4, kallikrein-related peptidase 4.

etiology, pathophysiology, and molecular mechanisms remain unknown. Further studies are required in order to fully understand and treat this disease.

\section{Acknowledgements}

This study would like to thank the following for providing financial support: The National Key Clinical Specialty Construction Programs of China (2014-2016), the Shanghai 'Non key-in-key discipline' Clinical medical centers (2014-2016), the Shanghai Rising-Star Program (A type) (grant no. 15QA1400800), the National Natural Science Foundation of China (grant no. 81572324) and the Science Foundation of Shanghai (grant no. 15ZR1404200). 


\section{References}

1. Honda S, Miyagi H, Suzuki H, Minato M, Haruta M, Kaneko Y, Hatanaka KC, Hiyama E, Kamijo T, Okada T and Taketomi A: RASSF1A methylation indicates a poor prognosis in hepatoblastoma patients. Pediatr Surg Int 29: 1147-1152, 2013.

2. Kingston JE, Herbert A, Draper GJ and Mann JR: Association between hepatoblastoma and polyposis coli. Arch Dis Child 58: 959-962, 1983

3. Ross JA: Hepatoblastoma and birth weight: Too little, too big, or just right? J Pediatr 130: 516-517, 1997.

4. Mamlok V, Nichols M, Lockhart L and Mamlok R: Trisomy 18 and hepatoblastoma. Am J Med Genet 33: 125-126, 1989.

5. Herman JG and Baylin SB: Gene silencing in cancer in association with promoter hypermethylation. N Engl J Med 349: 2042-2054, 2003.

6. Geiman TM and Robertson KD: Chromatin remodeling, histone modifications, and DNA methylation-how does it all fit together? J Cell Biochem 87: 117-125, 2002.

7. Reik W and Walter J: Genomic imprinting: Parental influence on the genome. Nat Rev Genet 2: 21-32, 2001.

8. Chow J and Heard E: X inactivation and the complexities of silencing a sex chromosome. Curr Opin Cell Biol 21:359-366, 2009.

9. O'Sullivan E and Goggins M: DNA methylation analysis in human cancer. Methods Mol Biol 980: 131-156, 2013.

10. Esteller M: Epigenetics in cancer. N Engl J Med 358: 1148-1159, 2008.

11. Kontos CK, Chantzis D, Papadopoulos IN and Scorilas A: Kallikrein-related peptidase 4 (KLK4) mRNA predicts short-term relapse in colorectal adenocarcinoma patients. Cancer Lett 330: 106-112, 2013.

12. Lose F, Srinivasan S, O'Mara T, Marquart L, Chambers S, Gardiner RA, Aitken JF; Australian Prostate Cancer BioResource, Spurdle AB, Batra J and Clements JA: Genetic association of the KLK4 locus with risk of prostate cancer. PloS One 7: e44520, 2012.

13. Haas JE, Feusner JH and Finegold MJ: Small cell undifferentiated histology in hepatoblastoma may be unfavorable. Cancer 92: 3130-3134, 2001

14. Johnson MR, Wang KS, Smith JB, Heslin MJ and Diasio RB Quantitation of dihydropyrimidine dehydrogenase expression by real-time reverse transcription polymerase chain reaction. Anal Biochem 278: 175-184, 2000.

15. Otte JB: Progress in the surgical treatment of malignant liver tumors in children. Cancer Treat Rev 36: 360-371, 2010.

16. Tiao GM, Bobey N, Allen S, Nieves N, Alonso M, Bucuvalas J, Wells R and Ryckman F: The current management of hepatoblastoma: A combination of chemotherapy, conventional resection, and liver transplantation. J Pediatr 146: 204-211, 2005
17. von Schweinitz D: Hepatoblastoma: Recent developments in research and treatment. Semin Pediatr Surg 21: 21-30, 2012.

18. Jia D, Dong R, Jing Y, Xu D, Wang Q, Chen L, Li Q, Huang Y, Zhang Y, Zhang Z, et al: Exome sequencing of hepatoblastoma reveals novel mutations and cancer genes in the Wnt pathway and ubiquitin ligase complex. Hepatology 60: 1686-1696, 2014.

19. Dong R, Jia D, Xue P, Cui X, Li K, Zheng S, He X and Dong K: Genome-wide analysis of long noncoding RNA (lncRNA) expression in hepatoblastoma tissues. PloS One 9: e85599, 2014.

20. Baylin SB and Ohm JE: Epigenetic gene silencing in cancer-A mechanism for early oncogenic pathway addiction? Nat Rev Cancer 6: 107-116, 2006.

21. Harada K, Toyooka S, Maitra A, Maruyama R, Toyooka KO, Timmons CF, Tomlinson GE, Mastrangelo D, Hay RJ, Minna JD and Gazdar AF: Aberrant promoter methylation and silencing of the RASSF1A gene in pediatric tumors and cell lines. Oncogene 21: 4345-4349, 2002

22. Zhang SQ, Cai B, Liu L, He YY, Yang YX and Wan XP: Kallikrein 4 overexpression in endometrial carcinoma and upregulation by estrogen via mitogen-activated protein kinase signal pathway. Int J Gynecol Cancer 19: 1377-1383, 2009.

23. Klokk TI, Kilander A, Xi ZJ, Waehre H, Risberg B, Danielsen HE and Saatcioglu F: Kallikrein 4 is a proliferative factor that is overexpressed in prostate cancer. Cancer Res 67: 5221-5230, 2007.

24. Xi ZJ, Kaern J, Davidson B, Klokk TI, Risberg B, Tropé C and Saatcioglu F: Kallikrein 4 is associated with paclitaxel resistance in ovarian cancer. Gynecol Oncol 94: 80-85, 2004.

25. Papachristopoulou G, Avgeris M and Scorilas A: Expression analysis and study of KLK4 in benign and malignant breast tumours. Thromb Haemost 101: 381-387, 2009.

26. Mangé A, Desmetz C, Berthes ML, Maudelonde T and Solassol J: Specific increase of human kallikrein 4 mRNA and protein levels in breast cancer stromal cells. Biochem Biophys Res Commun 375: 107-112, 2008

27. Doi A, Park IH, Wen B, Murakami P, Aryee MJ, Irizarry R, Herb B, Ladd-Acosta C, Rho J, Loewer S, et al: Differential methylation of tissue-and cancer-specific $\mathrm{CpG}$ island shores distinguishes human induced pluripotent stem cells, embryonic stem cells and fibroblasts. Nat Genet 41: 1350-1353, 2009

28. Irizarry RA, Ladd-Acosta C, Wen B, Wu Z, Montano C, Onyango P, Cui H, Gabo K, Rongione M, Webster M, et al: The human colon cancer methylome shows similar hypo- and hypermethylation at conserved tissue-specific $\mathrm{CpG}$ island shores. Nat Genet 41: 178-186, 2009.

29. Ogoshi K, Hashimoto SI, Nakatani Y, Qu W, Oshima K, Tokunaga K, Sugano S, Hattori M, Morishita S and Matsushima K: Genome-wide profiling of DNA methylation in human cancer cells. Genomics 98: 280-287, 2011. 